\title{
Editorial
}

\section{Yet Another Tool in the Armamentarium of Neuroanesthesiologist}

\author{
G.S. Umamaheswara Rao ${ }^{1}$ \\ ${ }^{1}$ Department of Neuroanaesthesia and Neurocritical Care, National \\ Institute of Mental Health and Neurosciences (NIMHANS), \\ Bangalore, India
}

J Neurosci Rural Pract 2019;10:569-570

Traditionally, drugs used to sedate the patients caused respiratory depression and airway obstruction. As a consequence of hypotension they produce, they also caused tachycardia. With the introduction of clonidine, a new class of drugs has come into clinical practice. Acting through central $\alpha-2$ receptor stimulation, they cause sedation and psychomotor indifference. These drugs produce sedation by decreasing the sympathetic nervous system activity. The result is a calm patient who can be easily aroused to full consciousness. Dexmedetomidine is a more potent $\alpha$-adrenergic agonist and has a shorter elimination half-life of 2 hours. It has sedative and analgesic sparing effects. Sedation with this drug is associated with reduced delirium and agitation, perioperative sympatholysis, cardiovascular stabilizing effects, and preservation of respiratory function. All these properties are desirable while anesthetizing a patient for transsphenoidal pituitary surgery.

Intraoperative hypertension is an important complication of transsphenoidal surgery. About 30\% of patients with acromegaly and $85 \%$ of patients with Cushing's syndrome have preoperative hypertension. Several techniques including various vasodilatory drugs, local anesthesia to the nasal mucous membrane, and even sphenopalatine ganglion block have been used to control this hypertension intraoperatively.

Dexmedetomidine has been used in neurosurgery for the past more than a decade. Several advantages have been documented with this drug in various studies. It has been shown to decrease intraoperative blood loss. Dexmedetomidine-lidocaine combination has been used for scalp infiltration in place of adrenaline-lidocaine combination. There is some experimental evidence to show its neuroprotective properties. ${ }^{1}$ It has been found to be a good sedative for monitored anesthesia care in combination with local block for chronic subdural hematoma drainage. ${ }^{2}$ Its analgesic property has been claimed to spare opioids during spine surgery. ${ }^{3}$ Dexmedetomidine-based sedation facilitated awake craniotomy without the need for urgent airway intervention and conversion of sedation to general anesthetic. This facilitates intraoperative neurophysiologic testing and real-time surgical decision making and thereby minimizing the risk of postoperative neurologic deficit. ${ }^{4}$ In one study in patients with traumatic brain injury, dexmedetomidine treatment attenuated early neurological dysfunction and brain edema. Further, dexmedetomidine attenuated posttraumatic inflammation, upregulated tight junction protein expression, and reduced secondary blood-brain barrier damage and apoptosis. Thus, it protected the brain against acute posttraumatic inflammatory response. ${ }^{5}$ In a study of 120 patients undergoing sevoflurane anesthesia for embolization of an intracranial aneurysm, one group received intravenous injection of $1.0 \mu \mathrm{g} / \mathrm{kg}$ dexmedetomidine for 15 minutes followed by maintenance with $0.3 \mu \mathrm{g} / \mathrm{kg} / \mathrm{h}$ until the end of surgery. Bispectral index monitoring revealed that dexmedetomidine-assisted anesthesia could shorten the recovery time of spontaneous breathing, time to eye opening, and time to laryngeal mask removal. The perioperative blood pressure and heart rate were stable, and postoperative delirium was less. ${ }^{6}$ Another study with 38 stroke patients who underwent endovascular thrombectomy under local anesthesia and dexmedetomidine consciousness sedation showed sedation by dexmedetomidine was effective during the endovascular treatment. Under dexmedetomidine sedation, endovascular thrombectomy was possible without significantly changing the patients' vital signs. ${ }^{7}$ Dexmedetomidine has been used to treat acute paroxysmal sympathetic overactivity. In spite of its effect on sympathetic nervous system, regional brain oxygenation and cerebral blood flow velocity are well preserved during dexmedetomidine and propofol sedation in patients undergoing treatment for movement disorders. ${ }^{8}$ Dexmedetomidine is effective in attenuating the heart rate and mean arterial pressure response to skull pin insertion before craniotomy. During surgery for movement disorders,

\author{
Address for correspondence \\ G.S. Umamaheswara Rao, MD, \\ Department of Neuroanaesthesia \\ and Neurocritical Care, National \\ Institute of Mental Health and \\ Neurosciences (NIMHANS), \\ Bangalore 560029, India \\ (e-mail: gsuma123@yahoo.com).
}

DOI https://doi.org/ 10.1055/s-0039-1700674 ISSN 0976-3147.
(C)2019 Association for Helping Neurosurgical Sick People

\section{License terms}

$($ () (1) $\Theta \circledast$ 
dexmedetomidine at low doses did not affect the microelectrode recording. Functional outcome was good and perioperative neurological events were less with dexmedetomidine compared with local anesthesia group. ${ }^{9}$ In a meta-analysis of 11 published randomized controlled studies involving 674 patients, dexmedetomidine reduced perioperative opioid consumption and postoperative pain intensity. ${ }^{10}$ Thus, across a spectrum of neurosurgical operations dexmedetomidine has been used successfully with various advantages.

Bala et $\mathrm{al}^{11}$ showed that dexmedetomidine administered during sevoflurane anesthesia caused less hemodynamic disturbance and rapid awakening of the patients undergoing transsphenoidal pituitary surgery. Given the pharmacological properties of the drug, that is not surprising. There are a few earlier papers reporting similar findings. One issue in this paper is that dexmedetomidine treatment has been compared with no treatment of the tachycardia and hypertension during septal dissection in the control group. The fact that hemodynamics were stable in the study group attests to the analgesic property of dexmedetomidine. Postoperative awakening was faster like in earlier studies. In any case, yet another study supports the use of dexmedetomidine to control the intraoperative hemodynamics and to help rapid recovery from anesthesia.

\section{Conflict of Interest}

None declared.

\section{References}

1 Lin N, Vutskits L, Bebawy JF, Gelb AW. Perspectives on dexmedetomidine use for neurosurgical patients. J Neurosurg Anesthesiol 2019;31(4):366-377

2 Srivastava VK, Agrawal S, Kumar S, Khan S, Sharma S, Kumar R. Comparative evaluation of dexmedetomidine and propofol along with scalp block on haemodynamic and postoperative recovery for chronic subdural haematoma evacuation under monitored anaesthesia care. Turk J Anaesthesiol Reanim 2018;46(1):51-56

3 Tsaousi GG, Pourzitaki C, Aloisio S, Bilotta F. Dexmedetomidine as a sedative and analgesic adjuvant in spine surgery: a systematic review and meta-analysis of randomized controlled trials. Eur J Clin Pharmacol 2018;74(11):1377-1389

4 McAuliffe N, Nicholson S, Rigamonti A, et al. Awake craniotomy using dexmedetomidine and scalp blocks: a retrospective cohort study. Can J Anaesth 2018;65(10):1129-1137

5 Wang D, Xu X, Wu YG, Lyu L, Zhou ZW, Zhang JN. Dexmedetomidine attenuates traumatic brain injury: action pathway and mechanisms. Neural Regen Res 2018;13(5):819-826

6 Tang CL, Li J, Zhang ZT, et al. Neuroprotective effect of bispectral index-guided fast-track anesthesia using sevoflurane combined with dexmedetomidine for intracranial aneurysm embolization. Neural Regen Res 2018;13(2):280-288

7 Nii K, Hanada H, Hiraoka F, Eto A, Mitsutake T, Tsutsumi M. Usefulness of consciousness sedation with dexmedetomidine and pentazocine during endovascular treatment for acute stroke. Neurol Med Chir (Tokyo) 2018;58(2):79-84

8 Farag E, Kot M, Podolyak A, et al. The relative effects of dexmedetomidine and propofol on cerebral blood flow velocity and regional brain oxygenation: a randomised noninferiority trial. Eur J Anaesthesiol 2017;34(11):732-739

9 Honorato-Cia C, Martinez-Simón A, Guridi J, Alegre M, Nuñez-Cordoba JM. Sedation during surgery for movement disorders and perioperative neurologic complications: an observational study comparing local anesthesia, remifentanil, and dexmedetomidine. World Neurosurg 2017;101:114-121

10 Liu Y, Liang F, Liu X, Shao X, Jiang N, Gan X. Dexmedetomidine reduces perioperative opioid consumption and postoperative pain intensity in neurosurgery: a meta-analysis. J Neurosurg Anesthesiol 2018;30(2):146-155

11 Bala R, Chaturvedi A, Pandia MP, Nithal PK. Intraoperative dexmedetomidine maintains hemodynamic stability and hastens postoperative recovery in patients undergoing trans-sphenoidal pituitary surgery. J Neurosci Rural Prac 2019;10(4):599-605 